\title{
K2: Background Survey - the search for undiscovered transients in Kepler/K2 data
}

\author{
R. Ridden-Harper, ${ }^{1,2,3 \star ~ B . ~ E . ~ T u c k e r, ~}{ }^{1,2,4}$ M. Gully-Santiago, ${ }^{5}$ G. Barentsen, ${ }^{5}$ \\ A. Rest, ${ }^{3,6}$ P. Garnavich, ${ }^{7}$ E. Shaya ${ }^{8}$ \\ ${ }^{1}$ Research School of Astronomy \&S Astrophysics, Mount Stromlo Observatory, The Australian National University, \\ Cotter Road, Weston Creek, ACT 2611, Australia \\ ${ }^{2}$ The ARC Centre for All-Sky Astronomy in 3-Dimensions (ASTRO3D) \\ ${ }^{3}$ Space Telescope Science Institute, 3700 San Martin Drive, Baltimore, MD 21218, USA. \\ ${ }^{4}$ The National Centre for the Public Awareness of Science, The Australian National University, \\ Canberra, ACT 2601, Australia \\ ${ }^{5}$ Bay Area Environmental Research Institute, P.O. Box 25, Moffett Field, CA 94035, USA. \\ ${ }^{6}$ The Johns Hopkins University, Baltimore, MD 21218, USA. \\ ${ }^{7}$ Physics Department, 225 Nieuwland Hall, University of Notre Dame, Notre Dame, IN 46556, USA. \\ ${ }^{8}$ Astronomy Department, University of Maryland, College Park, MD 20742-2421, USA.
}

Accepted XXX. Received YYY; in original form ZZZ

\begin{abstract}
The K2 mission of the Kepler Space Telescope offers a unique possibility to examine sources of both Galactic and Extra-galactic origin with high cadence photometry. Alongside the multitude of supernovae and quasars detected within targeted galaxies, it is likely that Kepler has serendipitously observed many transients throughout K2. Such events will likely have occurred in background pixels, coincidentally surrounding science targets. Analysing the background pixels presents the possibility to conduct a high cadence survey with areas of a few square degrees per campaign. We demonstrate the capacity to independently recover key K2 transients such as KSN 2015K and SN 2018oh. With this survey, we expect to detect numerous transients and determine the first comprehensive rates for transients with lifetimes $\leq 1$ day.
\end{abstract}

Key words: surveys - supernovae: general - gamma-ray burst: general

\section{INTRODUCTION}

Launched in 2009, the Kepler Space Telescope (Kepler) allowed for high precision and high cadence stellar photometry (Basri et al. 2005), with the goal of detecting thousands of exoplanets (Batalha 2014), until the failure of two reaction wheels led to a new mission profile $-K 2$. The $K 2$ mission extended Kepler observations along the ecliptic, presenting the opportunity to apply high precision and cadence photometry to a multitude of galactic and extragalactic sources (Howell et al. 2014).

The reduced telescope stability present in K2 affords it a lower photometric precision than the original Kepler mission. Despite the drop in photometric precision, K2 campaigns C01 to $\mathrm{C} 19$ were successful in obtaining unparalleled photometric data that lead to among other things, the detection of new exoplanets (e.g., Pearson et al. 2018), numerous supernovae (SN) (e.g., Garnavich et al. 2016; Olling et al. 2015; Rest et al. 2018; Dimitriadis et al. 2019; Shappee et al.

^ E-mail: ryan.ridden-harper@anu.edu.au
2019; Li et al. 2019), and variability in quasars (Aranzana et al. 2018).

The high cadence K2 observations make it possible to study short-duration structures in supernovae light curves and detect short-duration transients. Analysis of $K^{2}$ data has shown potential evidence for SN Ia shock interaction, as described in Kasen (2010), in at least one SN Ia, SN 2018oh (Dimitriadis et al. 2019), however, not in some other SN Ia (Olling et al. 2015). K2 has also shown evidence of shock breakouts in core-collapse supernovae (CCSN) (Garnavich et al. 2016), and lead to the discovery of KSN 2015K, a rapid transient that is thought to be powered by stellar ejecta producing a shock in the circumstellar medium (Rest et al. 2018). Short cadence observations were crucial to these results and offer the possibility to analyse a time domain that has previously been technically infesable.

All known K2 transients were detected through targeted observations of pre-selected galaxy targets, however, this may not be all the transient events detected with Kepler. As seen in Barclay et al. (2012) and Brown et al. (2015) it is possible that Kepler has serendipitously detected numerous 
events in background or sky pixels, such as super-outbursts of dwarf novae. Such background events will likely be faint and will require an analysis that will be heavily influenced by detector noise and telescope stability.

The high cadence observations of Kepler, present a unique possibility to probe a new parameter space unavailable to previous transient surveys. Current transient surveys cover larger areas than Kepler, however, they have longer cadences. The Pan-STARRS Medium Deep Survey had an nominal cadence of 3 days in any given filter to a depth of $\sim 23.3 \mathrm{mag}$ (Rest et al. 2014; Chambers et al. 2016); ZTF surveys the the sky North of $\delta=-31^{\circ}$ every 3 nights to $20.4 \mathrm{mag}$, through the public survey (Bellm et al. 2019); ASAS-SN covers the entire sky to $\sim 17 \mathrm{mag}$, at a nominal cadence of a few days (Kochanek et al. 2017); and ATLAS covers the sky North of $\delta=-30$ every two nights to 20.2 mag (Tonry et al. 2018). Previous surveys, such as the SNLS, ESSENCE, and SDSS-II also had a cadence of a few days, before taking into consideration bad weather, so would typically miss transients that evolve on timescales of $\sim$ days (Sullivan et al. 2011; Kessler et al. 2009; Miknaitis et al. 2007). Although these surveys sample volumes much larger, the comparatively high cadence observations of Kepler/K2 mean its legacy data can provide a unique wide-field transient survey.

A worldwide push is being made to extend surveys into high cadences to explore a new time domain for transients. Andreoni et al. (2020) present one such effort, known as Deeper, Wider, Faster (DWF), which coordinates multimessenger observations from 40+ telescopes for short 1 hour campaigns, with a cadence of 1.17 minutes. From this unique data set, an upper-bound on extragalactic fast transients was found to be $R_{e F T}<1.625 \mathrm{deg}^{-2} \mathrm{~d}^{-1}$. Although Kepler has a longer cadence of 30 minutes, a field is examined for $\sim 74$ days. Similarly, the TESS mission is an ongoing space-based mission, with 30-minute cadence and a wider field, albeit a lower sensitivity than Kepler / K2 (Ricker et al. 2015). Sharp et al. (2016) and Ridden-Harper et al. (2017) present a future high altitude balloon-based telescope system that aims to explore the time domain of days at ultraviolet wavelengths.

Marshall et al. (2017) presents the case for high cadence observations, highlighting the unique short duration events expected to be seen at Kepler-like cadences, such as GRB afterglows, other relativistic events, and supernovae shocks. As the time domain at the timescale of hours is relatively unexplored for transients, there is potential to discover exotic new transients and provide well-defined rates for current and upcoming high cadence transient surveys. Although $\mathrm{Ke}$ pler / K2 data can probe this parameter space, the classification of transients will be particularly challenging, since only observation in the Kepler filter will be available for most candidates.

Although challenging, examining every pixel in K2 data presents an opportunity to conduct a unique, high cadence survey over an appreciable volume. With this volume, Kepler can be used to examine expected rates of exotic short duration events, such as binary neutron star mergers, similar to the analysis Scolnic et al. (2018) conducted on existing survey data.

In this paper, we present the details of the "K2: Background Survey (K2:BS)" along with examples of positive detections. A companion paper Ridden-Harper et al. (2019) presents the analysis of KSN-BS:C11a, the first transient discovered in K2:BS. The analysis method is presented in $\S 3$, followed by the survey characteristics in $\S 4$ and example detections of known objects in $\S 5$. In $\S 6$ we present the extragalactic volume surveyed and the expected detection rates for an assortment of transients in $K 2$.

\section{$2 \quad K E P L E R / K 2$ DATA}

The Kepler / K2 data provides high cadence photometry on a wide range of targets. In each of the 19 campaigns between 50-100 targets were observed in short cadence mode with frames every $\sim 1$ minute, while between 10,000-20,000 targets were observed in long cadence mode, with frames every 30 minutes $^{1}$. In this paper, we will focus on data from the long cadence mode, however, this analysis technique is also applicable to short cadence data. Observation campaigns nominally lasted $\sim 80$ days, with some campaigns shortened, due to technical difficulties.

Kepler had a $116 \mathrm{deg}^{2}$ field-of-view (FOV), with a plate scale of $4^{\prime \prime}$ pixel $^{-1}$. Due to memory constraints, the entire Kepler FOV could not be telemetered so instead, science targets were pre-selected each observing campaign and allocated a pixel mask that extended several pixels around the science target, both for background subtraction and to account for telescope drift. These pixel masks are known as target apertures in the downloaded data cube, the Target Pixel Files (TPFs), and all targets have unique identifiers, known as the EPIC number for K2 (Huber et al. 2016) and KIC number for Kepler (Brown et al. 2011). As the TPFs record both spatial and temporal information they are the data we analyse in this project.

A key difference between $K 2$ data and that of the original Kepler mission is the poor telescope stability. Following the failure of 2 reaction wheels, leaving Kepler with only 2 functional reaction wheels, the $K 2$ mission was developed, using solar pressure on the solar array, in conjunction with the two remaining reaction wheels (Howell et al. 2014). This configuration was subject to drift up to $\sim 1$ pixel $\left(4^{\prime \prime}\right)$ every 6 hours, so the pointing was corrected by thruster firings every 6 hours, and are recorded as quality flags in the TPF. The drift is recorded in the TPF through two displacement parameters labelled POS_CORR, which give the local image motion, calculated from fitting motion polynomials to the centroids of bright stars in each detector channel (DAWG (Data Analysis Working Group) 2012). The POS_CORR values give a valuable reference to telescope stability throughout campaigns, with the total image displacement given by $D^{2}=$ POS_CORR $1^{2}+$ POS_CORR 22 .

All K2 fields are pointed along the ecliptic, enabling a range of galactic and extra-galactic fields to be observed. A consequence of pointing along the ecliptic is that a large number of asteroids cross through the K2 fields and contaminate data.

${ }^{1}$ https://keplerscience.arc.nasa.gov/data-products.html 


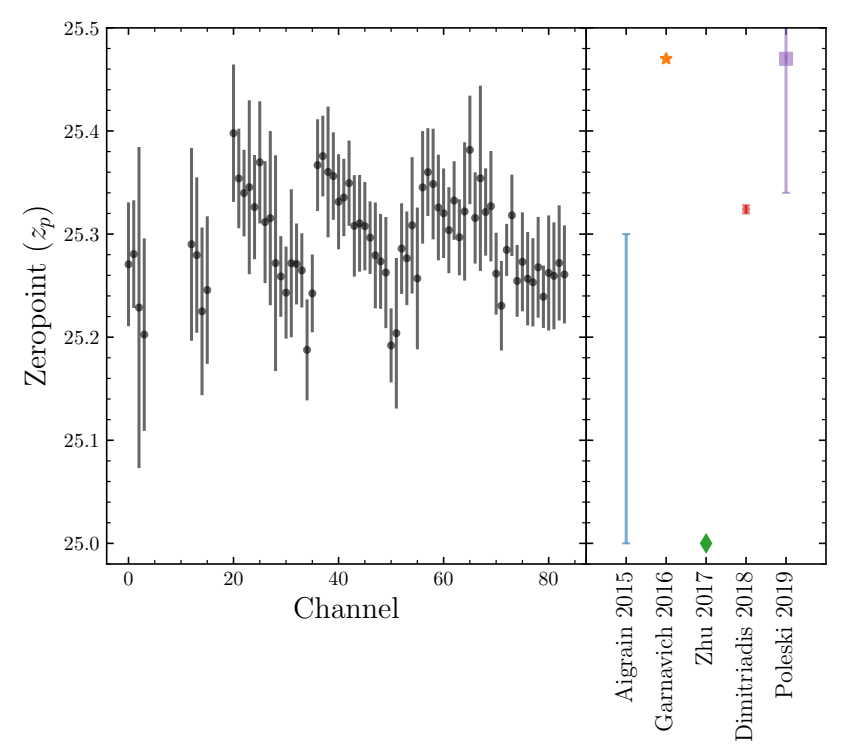

Figure 1. Left: Zeropoints we calculate for each Kepler/K2 channel, using stars from Campaigns C01, C06, C12, C16, and C17. Right: A selection of Kepler/K2 zeropoints taken from Aigrain et al. (2015), Garnavich et al. (2016), Zhu et al. (2017), Dimitriadis et al. (2019), and Poleski et al. (2019). The complete list of zeropoints are presented in Table A1, and Table A2.

\subsection{Kepler/K2 magnitudes and zeropoints}

We convert Counts, $C$, observed by Kepler to AB magnitudes, or $K_{p}$, according to

$$
K_{p}=-2.5 \log (\mathrm{C})+\mathrm{z}_{\mathrm{p}}
$$

where $z_{p}$ is the zeropoint. Since we analyse data across the entire Kepler/K2 field of view, we calculate the zeropoint independently for each of the 84 readout channels. This approach accounts for variability that may occur between the channels.

We calculate the $z_{p}$ for each channel by combining the $K_{p}$ magnitudes defined in the Ecliptic Plane Input Catalog (Huber et al. 2016) with the de-trended PDC EVEREST light curves (Luger et al. 2016) for all observed stars. We cut all stars with light curve variability $>1 \%$, and then perform a $3 \sigma$ sigma clip to remove outliers. Finally, we calculate the zeropoint for each channel using Eq. 1 and average across campaigns $\mathrm{C} 01, \mathrm{C} 06, \mathrm{C} 12, \mathrm{C} 14, \mathrm{C} 16$, and $\mathrm{C} 17$. The zeropoints for each channel are shown in Fig. 1 with values listed in Table A1.

We find that the zeropoints vary up to $0.2 \mathrm{mag}$ across the channels. When compared to zeropoints derived by others, we find that our zeropoints are consistent, with the exception of Zhu et al. (2017), which defines $z_{p}=25$. RiddenHarper et al. (prep) builds on this work to establish Kepler/K2 zeropoints that are comprehensively calibrated using Pan-STARRS photometry.

\section{METHODS}

The unique dataset from $K 2$ presents a number of challenges for event detection. Spacecraft drift, throughout K2 data, presents the largest challenge, requiring special treatment. Conventional methods such as image subtraction proved to be ineffective with the K2 data. The motion of up to $\sim 4^{\prime \prime}$ in K2 images resulted in poor subtractions with a median image. To counteract this we trialled subtracting images with similar displacements. While it achieved cleaner subtractions, it was not applicable to all images and introduced temporal biases in event detection. As these subtraction methods failed to be generally applicable, we developed new methods for event detection which still contain elements of conventional image subtraction.

We developed two methods to identify events, lasting from 1.5 hours to tens of days. One method can detect short events, with lifetimes $<10$ days, by assuming that the transient will be significantly brighter than background scatter; while the other method can detect long events with lifetimes $>10$ days, through heavy smoothing of the light curves and careful reference frame selection. The combination of these two methods successfully recovers short events, like asteroids and stellar flares, and long events such as supernovae.

The following section will describe the main data manipulation methods, followed by a detailed description of the detection methods. The analysis presented here is applicable to all Kepler, K2, and TESS data.

\subsection{Science target mask}

Each TPF contains science targets, other persistent sources and background pixels. To assist in event sorting, we identify each source and its extent. Identify the sources with a science target mask allows us to determine if events are associated with known objects, such as stars or galaxies.

We create the science target mask using a median frame with the following method. The median frame is generated by averaging a set of successive frames that have a total displacement less than 0.2 pixels from nominal telescope pointing. A sigma cut is performed on the median frame, where all pixels that are brighter than median $+\sigma$ of all pixels in the median frame are added to the science target mask. The previous sigma cut is repeated with the newly defined science target mask applied to the median frame, all pixels identified in this sigma cut are added to the science target mask.

To prevent transients from being included in the science target mask, we create two masks, one at the start and another at the end of the campaign. All science target mask pixels that appear in both the start and end masks are included in a final science target mask. Transients that can be identified by K2:BS must be shorter than the K2 campaign duration ( $\sim 80$ days), so this method ensures that detectable events aren't included in the science mask.

The large $\left(4^{\prime \prime}\right)$ pixels of Kepler make is possible that multiple sources blend together into a single mask. In an effort to separate these possible science targets, we separate the science target mask into multiple masks, through a watershed algorithm (Barnes et al. 2014). All of the science targets are checked against NASA/IPAC Extra-galactic 
Database (NED) ${ }^{2}$ and the Simbad (Wenger et al. 2000) database, to identify the object within each science target.

\subsection{Telescope drift correction}

During $K 2$ the telescope drift introduced significant instrumental artefacts to the data. During campaigns, Kepler drifts to a maximum of $\sim 1$ pixel from nominal telescope pointing, over $\sim 6$ hours. For bright targets, this effect is often negligible and can be offset with the motion correction tools present in Pyke (Still \& Barclay 2012; Vinícius et al. 2017), Lightkurve (Lightkurve Collaboration et al. 2018), K2SFF (Vanderburg \& Johnson 2014), and EVEREST (Luger et al. 2016).

The techniques used with K2SFF and EVEREST were developed to primarily correct stellar light curves, that don't feature strong variability. As a result these techniques fail to correct the background motion, without removing the transient signal. The Lightkurve PRF photometry tool can correct for telescope motion, while preserving transient signal, however, PRF photometry currently requires tailoring priors, such as position and flux, for each object in the TPF, and has a long computation time. These issues make PRF photometry infeasible for this analysis. As no existing detrending methods are suitable for a transient search, we develop a method to fit and remove motion induced noise for each pixel.

The telescope drift results in bright sources to periodically contaminating neighbouring pixels, creating discontinuous light curves. The impact that the telescope drift has on a light curve can be seen with the blue points in Fig. 2 . These motion induced signals increase the detection threshold and can masquerade as real transient signals, so it is critical that they are corrected.

Our data reduction method relies on thruster resets, which are identified through the quality flag 1048576. Utilising the thruster resets, we employ a data correction method that analyses each segment between thruster resets. The procedure acts on every pixel individually, altering the data with the following steps:

(i) All images/frames that have $<0.3$ pixel displacement in a campaign are identified. ${ }^{3}$

(ii) A $1 \mathrm{D}$ spline is fitted to the flux values of the image with the least displacement between thruster resets of the previously identified frames for each pixel.

(iii) The 1D splines are subtracted from each of the pixel light curves for the entire campaign, leaving residual flux that is largely the product of telescope motion.

(iv) The residual light curve is broken into segments defined by thruster resets.

(v) To avoid real transients from being removed in the motion correction, we perform a $2 \sigma$ sigma clip on the data.

2 The NASA/IPAC Extra-galactic Database (NED) is operated by the Jet Propulsion Laboratory, California Institute of Technology, under contract with the National Aeronautics and Space Administration

3 This choice is made based on data quality and the number of observations available at high precision.

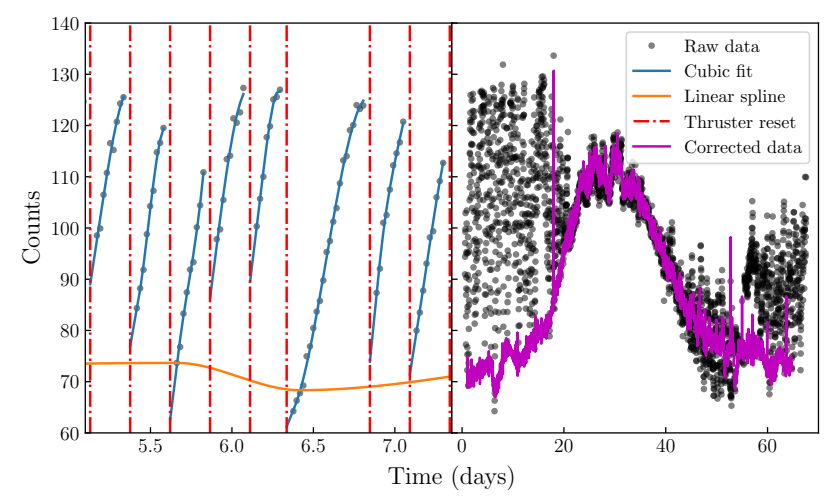

Figure 2. An example of the motion correction procedure on a pixel containing SN 2018ajj (Smith et al. 2018). Left: The components of the fitting procedure are shown: the thruster resets (red dashed line) divide the light curve into segments; a linear spline (orange line) is fit to the most stable points in each light curve segment; a cubic polynomials (blue lines) are fit to the raw data (black points). Right: The full light curve is shown for SN 2018ajj, with raw data (black points), and the motion corrected data (magenta line). This process dramatically reduces noise induced by telescope motion.

(vi) For each residual light curve segment, we fit a cubic polynomial through linear regression with scikit-learn (Varoquaux et al. 2015).

(vii) The cubic polynomial fits are subtracted from the residual flux calculated in step iii.

(viii) Finally the the $1 \mathrm{D}$ spline is added back to the data, resulting in motion corrected data.

This procedure is applied to all pixels, where the correction is most prevalent in pixels near the science target, or field stars. A dramatic example of this correction can be seen in Fig. 2, where through this motion correction we successfully reconstruct the light curve of SN 2018ajj (Smith et al. 2018). No extrapolation is included in this method, so the data becomes truncated to the first and last instance where the pointing accuracy is $<0.3$ pixels.

We find this procedure is successful at removing almost all motion artefacts from pixel light curves. The only residual artefacts that have been encountered are ones that persist between multiple thruster resets. Although we do not remove these trends, the false positives they produce in the event detection are removed through vetting steps discussed in $\S 3.3$.

\subsection{Short event identification ( $<10$ days)}

The core aspect of K2:BS is identifying real events from noise or other contaminants (e.g. asteroids). For each campaign every pixel is analysed independently to identify a potential event and an associated event time. A detection limit is calculated for each pixel as Limit $=$ median $+3 \sigma$ from all images. This cut-off value sets the magnitude limit for each pixel. Due to telescope drift, pixels close to science targets or stars have brighter limits than pixels with large separations. This highly variable level of counts, as seen in the magnitude limits shown in Fig. 7, is the motivation for analysing pixels individually. 
The process of identifying potential events utilises Boolean arrays and indexing. The $K 2$ target pixel file flux is converted to a Boolean array, conditioned on pixels having counts greater than the aforementioned limit. To avoid anomalous detections based on spacecraft operation all pixels in frames that coincide with nonzero quality flags, indicating anomalous behaviour, are set to false. Residual telescope motion and noise can cause false breaks in the Boolean array, as the light curve may periodically drop below the detection limit. These breaks will produce false event durations and may lead to transients being processed incorrectly and lost. To prevent noise from truncating event duration the Boolean detection array is smoothed by an iterative process of convolving each pixel, through time, with a 1d kernel of zeros with ones at the start and end positions. The smoothing process iterates from a length 9 kernel, to a length 3 kernel, with each iteration acting on the product from the previous step. The convolution process is as follows,

$$
\begin{aligned}
C & =D * k \\
A(C) & = \begin{cases}1, & \text { if } C=2 \\
0, & \text { if } C<2\end{cases} \\
R & =D+A(C),
\end{aligned}
$$

where $D$ is the initial Boolean detection array, $k$ is the $1 \mathrm{~d}$ smoothing kernel described above, $A(C)$ is the construction of a new Boolean array from, $C$, the convolved array, and $R$ is the final smoothed Boolean array used for detection. An example of this process with a length 3 kernel is shown in Fig. 3.

We use the smoothed Boolean array to identify candidate events with durations longer than a chosen baseline. The event identification process analyses each pixel independently and operates as follows:

(i) We identify the indices of all False values in the Boolean array.

(ii) The difference between neighbouring False indices is calculated.

(iii) Differences that are greater than a predefined baseline are selected as candidate events.

(iv) The start and end times of the candidate event are recorded along with the pixel position.

we set the minimum length to be 3 frames, or 1.5 hours, this requirement avoids detecting spurious noise or cosmic rays. This method proves successful in identifying transients that evolve rapidly over 1.5 hours to $\sim 10$ days.

Many of the potential events selected are false detections and contamination from known variable sources, that must be removed by subsequent checks. Sources of contamination can either be astrophysical (e.g., variable stars, asteroids), or instrumental (e.g., residual telescope motion). We impose a multi stage vetting procedure to limit false detections.

First we match all coincidental events. For each candidate event we collapse the Boolean detection array along the time axis from the beginning to the end of the candidate events duration. We then construct a Boolean mask

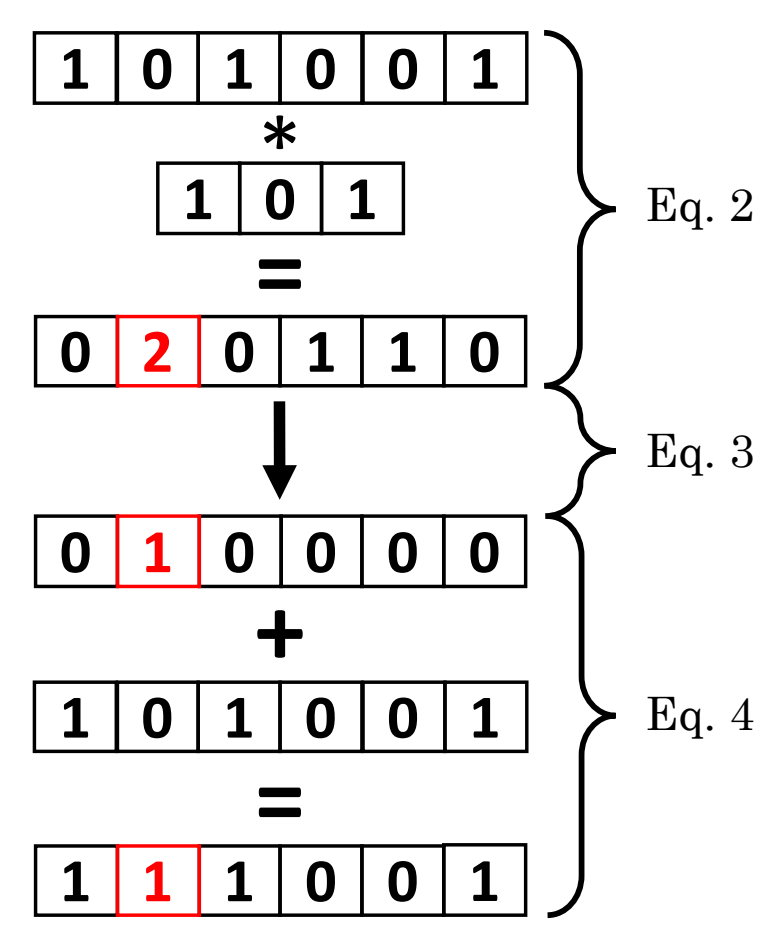

Figure 3. An example Boolean detection array being smoothed by a length 3 kernel. The smoothing prevents events from being truncated and rejected due to pixel count drops from instrument error.

array and set the pixel position of the candidate event to be True. The mask is then iterative convolved with a $3 \times 3$ True array and any True elements that appear in both collapsed Boolean array and the convolved mask are added to the mask, for the next iteration. This process terminates when no new pixel is added to the Boolean mask. Candidate events that are included in this new mask are merged into a single candidate.

Following the event matching procedure we vet candidates based on their the uniqueness. We smooth the light curve by with a Savitzky-Golay filter, setting the width to be twice the event duration. All peaks in the light curve are then identified using the Scipy find_peaks algorithm, which identifies local maxima through neighbour comparisons, and we condition on the peaks. We assume a real event will be significant and unique, so candidate events are rejected if: 1) the largest peak occurs outside the identified event time; 2) all peaks within a time frame less than twice the event duration from the candidate event must be less than $80 \%$ the brightness of the maximum peak. We find that in areas of prolonged poor pointing, a forest of similar peaks emerge, which condition 2 is instrumental in vetting.

Although these conditions are successful in eliminating almost all false detections, they introduce complexity into the true limiting magnitude. A thorough analysis of the magnitude limits for each pixel will be presented in future work. 


\subsection{Long event identification ( $>10$ days)}

This method closely follows that of the short detection method, however, greater care is taken in the construction of the pixel limits. As the K2 campaigns nominally last for $\sim 80$ days, long transients, such as supernovae (SN) can strongly impact the light curve throughout the campaign, raising the detection limit to a point where the transient is no longer detectable. In order to construct an accurate limit we must examine trends in the light curve.

First, we smooth the light curve with a Savitzky-Golay filter with a window of 5 days. Although this process truncates the light curve by 10 days, it removes the signatures of short events (e.g., asteroids) from the light curve. We then identify the largest peak in the data using the Scipy find_peaks algorithm (Virtanen et al. 2019), and break the light curve into 3 zones: the event zone, extending 10 days before to 25 days after the peak; and zones 1 and 2, before and after the event zone respectively. As some transients, such as SN can have long declines, we compare the light curve properties of zones 1 and 2 with the following;

$$
\mu_{2}>\mu_{1}+3 \sigma_{1},
$$

where $\mu_{1,2}$ are the light curve means of zones 1 and 2 , and $\sigma_{1}$ is the standard deviation of zone 1 . If this condition is met then only zone 1 is used to calculate the limit, otherwise, both zones are used to calculate the limit. As with the short detection method, this limit is determined for each pixel individually as Limit $=$ median $+3 \sigma$.

With limits for each pixel, candidate long events are selected through a similar process to short events. Without smoothing the Boolean array, we locate events by the positions of False values. In this case we require the event duration to last longer than 10 days. For these events, we assume that they are unique and have well behaved peaks, as such candidate events are vetted by the following conditions: 1) the largest peak occurs inside the identified event time; 2) the peak can be well fit by a $3^{\text {rd }}$ degree polynomial and poorly fit by a $1^{\text {st }}$ degree polynomial, requiring the coefficient of determination $\left(R^{2}\right)$ to be $>0.95$ and $<0.5$ respectively.

\subsection{Variable stars}

Bright variable stars and their associated overflow into columns of pixels can lead to false detections. These false detections are mitigated by checking the pixels neighbouring the event pixel, if one of the neighbours contain $>100,000$ counts the event is considered false and discarded. This value is chosen as it encompasses the apparent variability in the saturation level of the pixels. If the bleeding of the target occurs at a lower count rate than the cut-off, then it may also be contained in a "Probable" event category. This category is discussed further in $\S 3.7$.

\subsection{Asteroids}

Due to K2 observing along the ecliptic, there are many asteroids that pass through the data. As most asteroids remain in the TPF FOV for $<1$ day, they are predominantly detected by the short event method. The asteroid detection method

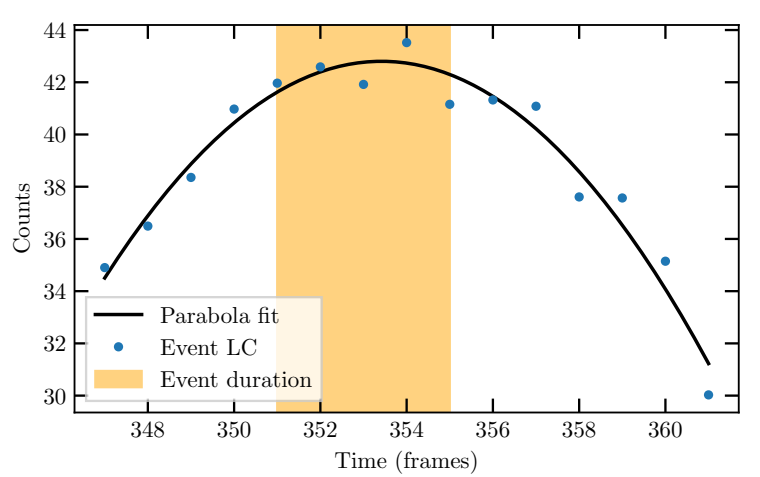

Figure 4. An example of a potential event identified as an asteroid in C01, EPIC 201735583. Since asteroids travel at a relatively constant speed through the aperture, the light curves they produce are largely parabolic. Thus parabolic light curves of short events are classified as asteroids.

utilises the fact that asteroids tend to move with a near constant speed through the TPF, thus they produce a parabolic light curve, as seen in Fig. 4. K2:BS identifies asteroids by fitting a parabola to frames \pm 2 from the brightest point in the potential event light curve. The generated parabola is then fitted and subtracted from the event light curve, if the residual is small then the potential event is categorised as an asteroid. All asteroids are recorded and can be examined independently. It is possible that real short transients may fall within the asteroid criteria, however, such events could be recovered at a later point following an analysis of asteroid-like events ${ }^{4}$.

\subsection{Event sorting}

For each object detected, we must search for a potential host or source of the event. To simplify visual vetting of candidate events, they are split into categories based on several event aspects such as duration, brightness, detection method, source type, and relation to masked objects.

Identifying the host of a potential event can follow different pathways. For each detection, we query NED and Simbad to identify potential sources or host galaxies. If the event occurs within the mask of a science target, the host is defined to be the science target, and the candidate event is sorted into the "In" category and subcategory based on the host type. Similarly, events that occur next to a science target mask are sorted into the "Near" category and host type subcategory. Candidate events that occur in the background pixel, not associated with a science target, are identified through querying the coordinates corresponding to the brightest pixel, and sorted into a category corresponding to the likely host type.

4 Although uncommon, some asteroids will move through an epicycle within the TPF. These asteroids do not have uniform motion, and so can be missed by the asteroid filter. We are currently working on adaptive masks that are capable of tracking asteroids. These masks would extract all asteroid information and separate asteroids from transients. 
If a candidate event's coordinates do not correspond to an object in NED or Simbad database, it is assigned to the "Unknown" category. This category often contains false detections form the $K 2$ electronic noise sources, however it can also contain previously unseen events and some of the most exciting events promised by this analysis. An example of a new object found through an "Unknown" classification can be found in Ridden-Harper et al. (2019).

\subsection{Event ranking}

As a further diagnostic to assist in the vetting of candidate events is a series of quality rankings. Each candidate receives ranking for the brightness, duration, mask size, and source/host type. These rankings, as outlined below, provide a way of sorting candidate events into prioritised lists, simplifying the vetting process.

- The brightness ranking is taken as the significance of the event. The significance is found by calculating the peak flux of the candidate event and comparing it to the median and standard deviation of the entire light curve, excluding points from two days before the candidate event starts, to ten days after it ends.

- The duration ranking is simply the calculated duration of the event in days.

- The mask size ranking is calculated from the number of pixels included in the candidate event mask. This ranking is normalised such that three or more pixels in a mask produce a maximum rank of 1 .

- The host ranking is based on classification given to the candidate event, from the NED object classification system. Objects that aren't of interest to this survey, such as stars, are ranked 0 , while objects of interest, such as galaxies, quasars, etc., and unknown objects, are ranked 1.

\subsection{Visual inspection}

If an event passes through the K2:BS conditions it is finally checked through visual inspection. K2:BS generates an event figure, video, and event positions. An example detection figure is shown in Fig. 5 for a short outburst from quasar [HB89] 1352-104. The top of the figure contains diagnostic information on the object and position; left shows the event light curve, with diagnostic information, such as thruster firings and quality flags, the background and nearest science target light curves, and the identified event duration in orange; right shows the reference image at the top and the brightest frame from the event at the bottom.

Selected frames from the corresponding event video for [HB89] 1352-104 are shown in Fig. 6. Left is the event light curve with the vertical red line showing the time stamp of the K2 frame shown on the right.

These figures, alongside candidate event sorting provide enough information to accurately assess the validity of a candidate event.

\section{SURVEY CHARACTERISTICS}

As this survey is using existing data, survey limits and characteristics are set by the original data, as discussed in $\S 2$.
Table 1. Number of background pixels, and the equivalent on sky area for $K_{2}$ extragalactic pointing campaigns.

\begin{tabular}{lccc}
\hline Campaign & Pixels & Area $\left(\mathrm{deg}^{2}\right)$ & Duration (days) \\
\hline C01 & 3894581 & 4.8 & 83 \\
C06 & 1957708 & 2.4 & 79 \\
C12 & 2120713 & 2.6 & 80 \\
C13 & 1645855 & 2.0 & 81 \\
C14 & 2095376 & 2.6 & 81 \\
C16 & 1408794 & 1.7 & 81 \\
C17 & 2590027 & 3.1 & 69 \\
\hline
\end{tabular}

Key survey parameters are the cadence, survey time, area, and depth. Operating with $K 2$ long cadence data fixes the cadence to 30 minutes and survey time per field to $\leq 80$ days. Events can only be detected if they are longer than the minimum event duration of 8 frames or 4 hours (set to prevent contamination from short scale noise or cosmic rays, and short enough to exhibit variation over a campaign, so that the long detection method can be successful.

Since two distinct detection methods are used that probe two different time domains, it is necessary to define the limits and recovery rates for both. Although the two methods target different time domains, there is an overlap for events with peaks or durations that last $\sim 2$ days. This overlap prevents detection gaps in the time domain between the two.

As both detection methods scan every pixel, they feature the same survey area. For this analysis, we will focus on extra-galactic pointing fields, which have the best chance at detecting transients. As seen in Tab. 1, the background pixels cover a significant area. C01 features the highest number of background pixels, due to each science target being assigned larger pixel masks. Although C16 is an extra-galactic field, the area of background pixels is significantly lower than that of the other due to the field containing the Beehive cluster, and the Earth.

\subsection{Magnitude limits}

K2:BS is limited to the area shown in Tab. 1, however, it has a highly variable magnitude limit. In this analysis the limiting magnitude is set by the detection limit that is imposed and discussed in $\S 3.3$ and 3.4. The interplay of the science target and telescope drift sets different magnitude limits, and therefore volumes, for each pixel. An example of the variable magnitude limit for a target pixel file can be seen in Fig. 7. In general, pixels close to the science target have brighter limits, than those further away. This limit provides a simple way to calculate the expected volumetric rate of events.

Due to the mission design of $K 2$, the majority of downloaded pixels do not contain a target, and have faint limiting magnitudes. The distribution of pixel limiting magnitudes are shown for $\mathrm{C} 06$ in Fig. 8, where $\sim 80 \%$ of pixels have limiting magnitudes $K_{p} \geq 18$, which are ideal for transient detection. Although some pixels report limiting magnitudes fainter than $K_{p}=22$, it is unlikely that such sensitivity can be achieved through this analysis. In all rate calculations we set the limiting magnitudes of pixels with $K_{p}>22$ to 22 .

Although the limit provided by K2:BS is representa- 

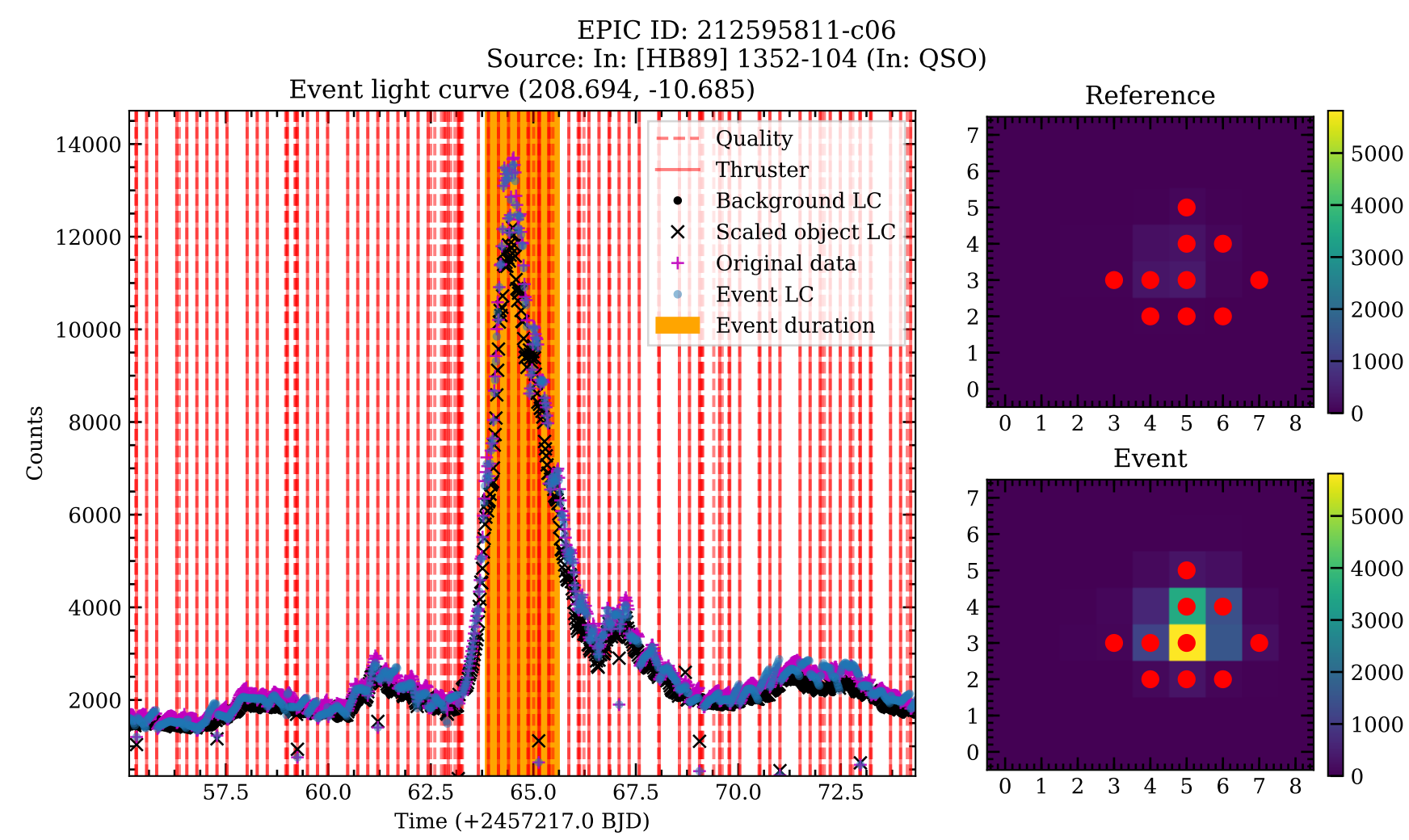

Figure 5. Example of a vetted, real event detected by K2:BS. This event was detected in K2 object 212595811, which is a short 3 day outburst from the quasar [HB89] 1352-104. During the outburst the apparent magnitude increases from $17.2 K_{p}$ to $15.1 K_{p}$. The light curve on the left presents the event light curve along with diagnostic information such as when a thruster reset occurs and quality flags. The sub-figures on the right show the reference and peak brightness event frame, with the event mask overlaid in red points.

tive of the expected limiting magnitude, the candidate event vetting process will introduce complexities. As discussed in $\S 3.3$ and 3.4 both the short and long detection methods undergo vetting to remove false detections. These checks have the potential to discard real transients if data quality is sub-optimal. We will present volumentric rates and a robust analysis of detection efficiency and contamination for a variety of key transients in a future work.

\section{DETECTED EVENTS}

The pilot program of K2:BS identified a number of known and undiscovered events. Initial runs have yielded promising results, by independently recovering key transients discovered in the Kepler Extra-galactic Survey, and recovering a superoutburst of a dwarf WZ Sge nova which is presented in Ridden-Harper et al. (2019). Here we will present example detections of known events, independently recovered by K2:BS, that we use as method verification.

Two examples of short transient events detected by K2:BS are variability in quasar [HB89] 1352-104, and KSN 2015K. During C06, quasar [HB89] 1352-104 experienced an outburst that lasted $\sim 3$ days, as seen in Fig. 5 . Variability pre- and post- outburst are also visible. The short transient KSN 2015K (Rest et al. 2018) was also recovered, as seen in Fig. 9. The position of the event was determined to within to within $4^{\prime \prime}$ of the accepted value, which is the resolution limit of Kepler. Both of these events are key examples of short events that may be detected by K2:BS.

K2:BS is also capable of detecting type Ia supernovae, such as SN 2018oh, as seen in Fig. 10. This event was detected through the long detection method. As well as detecting SN 2018oh, the excess light at rise is visible, as discussed in Dimitriadis et al. (2019); Shappee et al. (2019); Li et al. (2019).

\section{EXPECTED RATES}

The motivation for the K2:BS is to detect transient events in K2 data, so its useful to determine the likely number of events the survey should detect. Since each K2 campaign is unique, the survey area and therefore event rates vary between campaigns. In the following sections we present the expected detection rate for SN Ia and CCSN, gamma ray burst (GRB) afterglows, kilonova, and the fast-evolving luminous transients (FELTs) detected by PAN-STARRS1 (Drout et al. 2014). FELTs, kilonova, and GRB afterglows evolve rapidly, so are detectable by the short detection method and we therefore use rates from this method, while SN such as SN Ia and CCSN are detected through the long event detection method and we use respective rates.

There are few known types of transients that have life- 


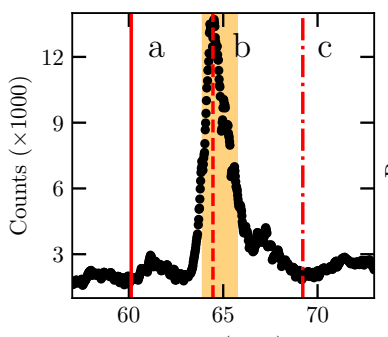

Time (days)
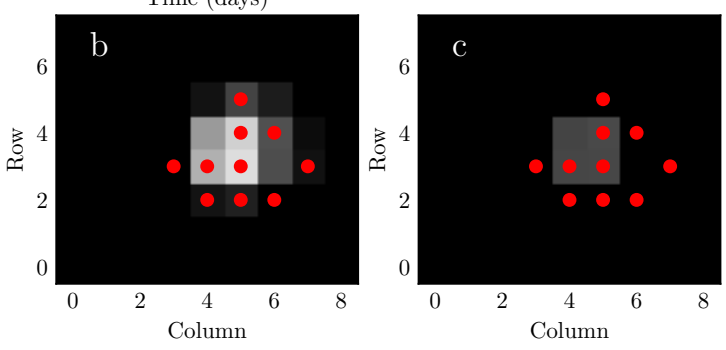

Figure 6. Example frames from the K2:BS event video for a short outburst from quasar [HB89] 1352-104. A simplified light curve is shown in the top left, the detected event is highlighted in orange. Panels $a, b$, and $c$ are K2 images corresponding to the times indicated by red lines in the light curve.

times on the order of days. As K2:BS will probe the $\sim 1$ day time domain, the results of this survey will be used to define rates for day to sub-day duration transients and presented in future work.

\subsection{Volumetric rates}

For K2:BS the volumetric rates are derived for each pixel and summed to reach the final rate. The motivation for this segmented approach is influenced by the large pixel size and, more importantly, the high variability in sensitivity between pixels. By knowing the magnitude limit per pixel we can calculate the volume, $V$, as follows,

$$
V=\frac{\theta}{3}\left(10^{\frac{1}{5}(m-M+5)}\right)^{3}
$$

where $\theta$ is the solid angle of a pixel in steradians, $m$ is the magnitude limit of a pixel, and $M$ is the absolute magnitude of the transient. As these calculations are dependent on an absolute magnitude, the volumes are model dependent.

For supernovae we use the $\mathrm{Li}$ et al. (2011) type Ia supernova rate of $(0.301 \pm 0.062) \times 10^{-4} \mathrm{Mpc}^{-3} \mathrm{yr}^{-1}$ and an absolute magnitude of -19.0 . For the kilonova rate we use $1540_{-1220}^{+3200} \mathrm{Gpc}^{-3} \mathrm{yr}^{-1}$ from Abbott et al. (2017), and take the absolute magnitude to be -16 , based off GW170817 light curves presented in Villar et al. (2017). The FELT rate is taken as $6400 \pm 2400 \mathrm{Gpc}^{-3} \mathrm{yr}^{-1}$ with an absolute magnitude range of -16.5 to $-20 K_{p}$ from Drout et al. (2014).

Since GRB light curves are highly model dependent, we will explore their rates in the following subsections.

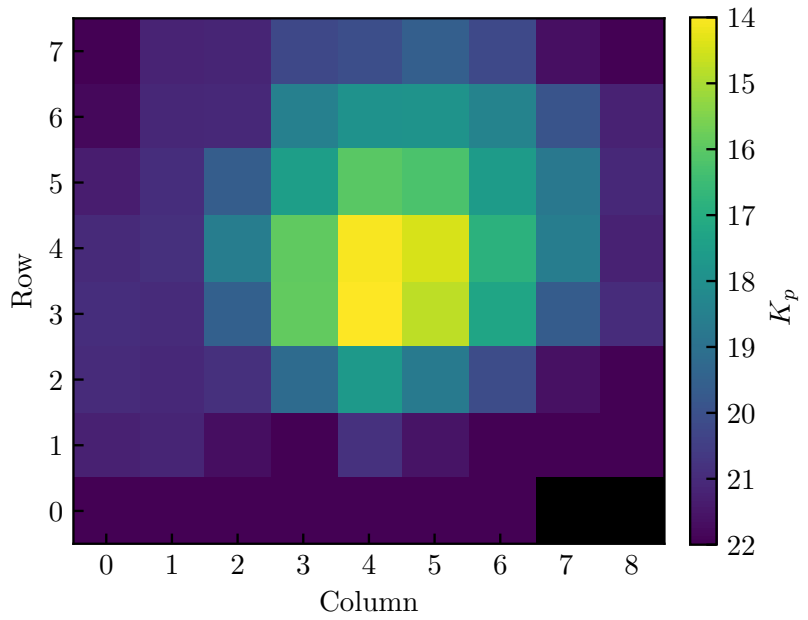

Figure 7. Magnitude limit for EPIC 212787678 from C17. Pixels near the original science target (centre frame) have a brighter limiting magnitude due to the science target's brightness, variability and residual telescope drift, with the pixels far from the target have a faint limiting magnitude. Each field has an associated magnitude limit from which the observed volume can be calculated.

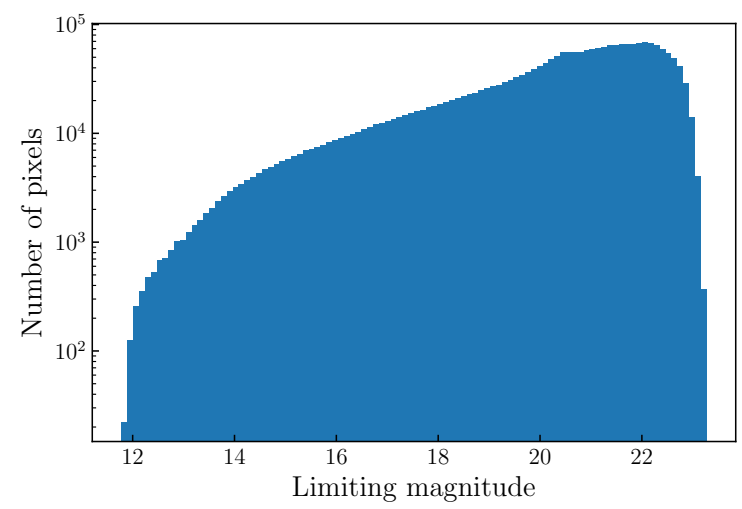

Figure 8. Distribution of pixel limiting magnitudes for C06. The high proportion $(\sim 80 \%)$ of pixels with faint limits $\left(K_{p} \geq 18\right)$ are ideal for transient searches.

\subsubsection{GRB rates}

For this analysis we use the afterglowpy package described in Ryan et al. (2019) for GRB afterglow light curves. In this exploratory case, we generate GRB afterglows using the afterglowpy top hat jet with the following set of fiducial GRB parameters; isotropic-equivalent energy, $E_{0}=10^{52} \mathrm{erg}$; halfopening angle, $\theta_{j}=3^{\circ}$ (Nicuesa Guelbenzu et al. 2012); circumburst density, $n=1.0 \mathrm{~cm}^{-3}$; energy equipartition factors of the electrons and magnetic field, $\epsilon_{e}=0.1$ and $\epsilon_{B}=0.01$ respectively.

The apparent magnitude of a GRB afterglow depends on both the viewing angle, $\theta_{\text {obs }}$, and the redshift, $z$. We calculate the apparent magnitude of the GRB afterglow independently varying $\theta_{\text {obs }}$ from $0-90^{\circ}$ in steps of $0.5^{\circ}$ and 


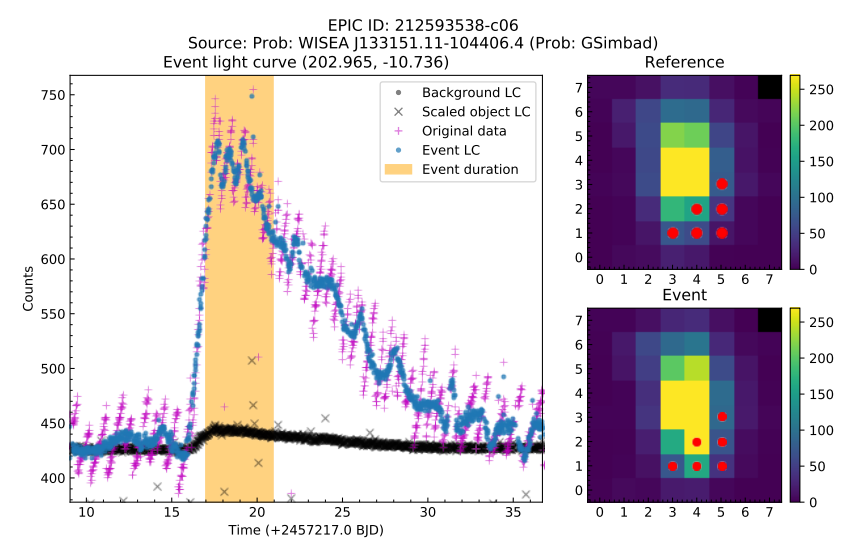

Figure 9. K2:BS independent detection of KSN 2015K, presented in Rest et al. (2018). This event was recovered through the "short" event detection method.

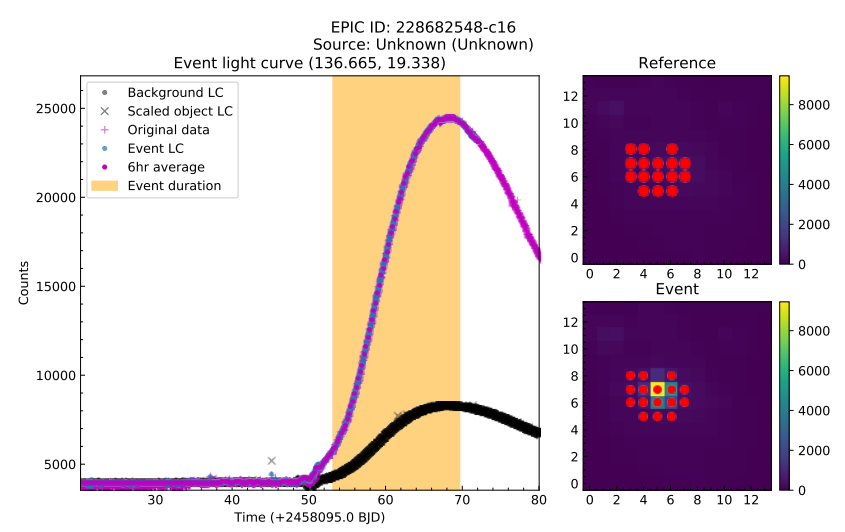

Figure 10. K2:BS independent detection of SN 2018oh, presented in Dimitriadis et al. (2019); Shappee et al. (2019); Li et al. (2019). This event was recovered with the "long" event detection method.

$z$ from $0.01-5$ in steps of 0.01 . By comparing the limiting magnitude of a pixel to the GRB magnitude array, we can determine the maximum $z$ a pixel could detect a GRB for a given viewing angle.

Following Guetta et al. (2005), we approximate the GRB formation rate with the star formation rate from Rowan-Robinson (1999);

$$
R_{G R B}=\rho_{0}\left\{\begin{array}{ll}
10^{0.75 z}, & z<1 \\
10^{0.75}, & z \geq 1
\end{array},\right.
$$

where $\rho_{0} \approx 33 h_{65}^{3} \mathrm{Gpc}^{-3} \mathrm{yr}^{-1}$ (Guetta et al. 2005). From Japelj \& Gomboc (2011) the number of GRBs per year is given by

$$
N=\int_{0}^{z_{\max }} \frac{R_{\mathrm{GRB}}}{1+z} \frac{d V}{d z} d z,
$$

where the co-moving volume element, $d V / d z$, is given by

$$
\frac{d V}{d z}=\frac{c}{H_{0}} \frac{4 \pi \chi^{2}(z)}{E\left(\Omega_{i}, z\right)}, \quad \chi=\frac{c}{H_{0}} \int_{0}^{z^{\prime}} \frac{d z^{\prime}}{E\left(\Omega_{i}, z^{\prime}\right)},
$$

and $E\left(\Omega_{m}, z\right)=\sqrt{\Omega_{m}(1+z)^{3}+\Omega_{\Lambda}}$. For these calculations we adopt a standard $\Lambda$ CDM cosmological model where $H_{0}=$ $72 \mathrm{~km} \mathrm{~s}^{-1} \mathrm{Mpc}^{-1}, \Omega_{m}=0.3$, and $\Omega_{\Lambda}=0.7$.

With the fiducial GRB light curves and occurrence rate, we calculate the expected number of detections. The number of detections are calculated per pixel, accounting for all possible viewing angles. In these calculations we take "On-Axis" GRBs to be $\theta_{\text {obs }} \leq \theta_{j}$ and "Off-Axis" GRBs, or Orphan Afterglows, to be $\theta_{o b s}>\theta_{j}$.

\subsection{Expected detection rates}

Tab. 2 shows the expected number of events from various $K 2$ extra-galactic campaigns. We find that it is unlikely that $K 2$ serendipitously observed any kilonova or GRBs during the extragalactic pointing campaigns, however, it is likely that K2 serendipitously observed many SN Ia and FELTs. Although the expected rates for SN Ia and FELTs appear high, they are likely overestimates, due to intricacies in the K2 field selections. The Kepler Extragalactic Survey (KEGS) survey will bias K2:BS towards observing galaxies, as most large galaxies were selected, while the star targets may bias K2:BS against galaxies. These subtle, but important, biasing factors will be incorporated into a follow-up paper where we present the comprehensive transient rates from K2:BS, following completion of the survey.

\section{CONCLUSIONS}

The Kepler K2 mission has provided a wealth of information on transient events. Through a directed survey of over 40,000 galaxies KEGS found a multitude of transients, including a rare fast transient KSN 2015K (Rest et al. 2018) and the potential detection of a SN Ia shock interaction in SN 2018oh (Dimitriadis et al. 2019; Shappee et al. 2019; Li et al. 2019). The transients detected by KEGS are not the only transients detected by Kepler, a number of events may have been detected serendipitously. The precedent on this was set by the discovery of a dwarf cataclysmic variable outburst in the original Kepler mission (Barclay et al. 2012).

Through the K2: Background Survey, we propose to analyse all pixels in K2 data for serendipitous transient detections. This survey will be blind, with final candidates vetted. The survey is not limited to specific types of transients, as all events that meet the detection threshold will be selected for final vetting. This unconstrained search, coupled with the rapid cadence of Kepler will allow us to search for transients in the time domain of hours, which is poorly understood.

This survey will cover $\sim 3 \mathrm{deg}^{2}$ per campaign, with a total area of $\sim 50 \mathrm{deg}^{2}$ through all K2 fields. The total area, combined with the depth of up to $22 K_{p}$ makes it likely that a number of unknown transients have been observed during K2. This is made evident by the first reported detection of a WZ Sge dwarf nova superoutburst presented in RiddenHarper et al. (2019), discovered by K2:BS.

The analysis method presented here is not only applicable to K2 data. As Kepler and TESS share the same data structure as K2, they too can be analysed through the background survey. The search strategies will also be more effective in Kepler and TESS data, due to the comparative 
Table 2. Volumetric rates for the extra-galactic pointing K2 campaigns for SN Ia, kilonovae, and FELTs. C01 has the largest rate, due to larger pixel masks around objects, and therefore more background pixels and the largest number of expected detections. Conversely C16, although an extra-galactic field, contains the Beehive cluster and Earth, which both severely reduce the sensitivity.

\begin{tabular}{lccccc}
\hline Campaign & SN Ia & $\begin{array}{c}\text { Kilonova (NS-NS) } \\
\left(\times 10^{-3}\right)\end{array}$ & $\begin{array}{c}\text { FELT } \\
\left(-16.5 K_{P} \rightarrow-20.0 K_{P}\right)\end{array}$ & $\begin{array}{c}\text { GRB } \\
\text { On-axis }\end{array}$ & Off-axis \\
\hline C01 & $12 \pm 2$ & $10_{-8}^{+20}$ & $0.08 \pm 0.02 \rightarrow 10 \pm 3$ & 0.14 & 0.03 \\
C06 & $7 \pm 1$ & $6_{-4}^{+11}$ & $0.05 \pm 0.01 \rightarrow 6 \pm 1$ & 0.08 & 0.02 \\
C12 & $7 \pm 2$ & $6_{-5}^{+12}$ & $0.05 \pm 0.01 \rightarrow 6 \pm 2$ & 0.08 & 0.02 \\
C13 & $6 \pm 1$ & $5_{-4}^{+9}$ & $0.04 \pm 0.01 \rightarrow 5 \pm 1$ & 0.06 & 0.01 \\
C14 & $8 \pm 2$ & $6_{-5}^{+13}$ & $0.05 \pm 0.01 \rightarrow 6 \pm 2$ & 0.08 & 0.02 \\
C16 & $5 \pm 1$ & $4_{-3}^{+8}$ & $0.03 \pm 0.01 \rightarrow 4 \pm 1$ & 0.05 & 0.02 \\
C17 & $9 \pm 2$ & $7_{-6}^{+15}$ & $0.06 \pm 0.02 \rightarrow 8 \pm 2$ & 0.10 & 0.02 \\
\hline
\end{tabular}

high stability. Following the analysis of K2, the background survey will run on these two additional data sets.

\section{ACKNOWLEDGEMENTS}

We thank Geoffrey Ryan for his assistance in implementing afterglowpy. This research was supported by an Australian Government Research Training Program (RTP) Scholarship and utilises data collected by the K2 mission. Funding for the K2 mission is provided by the NASA Science Mission directorate. We also make significant use of the NASA/IPAC Extragalactic Database (NED), which is operated by the Jet Propulsion Laboratory, California Institute of Technology, under contract with the National Aeronautics and Space Administration.

\section{REFERENCES}

Abbott B. P., et al., 2017, Phys. Rev. Lett., 119, 161101

Aigrain S., Hodgkin S. T., Irwin M. J., Lewis J. R., Roberts S. J., 2015, MNRAS, 447, 2880

Andreoni I., et al., 2020, MNRAS, 491, 5852

Aranzana E., Körding E., Uttley P., Scaringi S., Bloemen S., 2018, MNRAS, 476, 2501

Barclay T., Still M., Jenkins J. M., Howell S. B., Roettenbacher R. M., 2012, MNRAS, 422, 1219

Barnes R., Lehman C., Mulla D., 2014, Computers and Geosciences, 62, 117

Basri G., Borucki W. J., Koch D., 2005, New Astronomy Reviews, 49, 478

Batalha N. M., 2014, Proceedings of the National Academy of Science, 111, 12647

Bellm E. C., et al., 2019, PASP, 131, 068003

Brown T. M., Latham D. W., Everett M. E., Esquerdo G. A., 2011, AJ, 142, 112

Brown A., et al., 2015, AJ, 149, 67

Chambers K. C., et al., 2016, arXiv e-prints, p. arXiv:1612.05560

DAWG (Data Analysis Working Group) 2012, Kepler: A Search for Terrestrial Planets Kepler Archive Manual

Dimitriadis G., et al., 2019, ApJ, 870, L1

Drout M. R., et al., 2014, ApJ, 794, 23

Garnavich P. M., Tucker B. E., Rest A., Shaya E. J., Olling R. P., Kasen D., Villar A., 2016, ApJ, 820, 23

Guetta D., Piran T., Waxman E., 2005, ApJ, 619, 412

Howell S. B., et al., 2014, PASP, 126, 398

Huber D., et al., 2016, ApJS, 224, 2
Japelj J., Gomboc A., 2011, PASP, 123, 1034

Kasen D., 2010, ApJ, 708, 1025

Kessler R., et al., 2009, ApJS, 185, 32

Kochanek C. S., et al., 2017, PASP, 129, 104502

Li W., Chornock R., Leaman J., Filippenko A. V., Poznanski D., Wang X., Ganeshalingam M., Mannucci F., 2011, MNRAS, 412,1473

Li W., et al., 2019, ApJ, 870, 12

Lightkurve Collaboration et al., 2018, Lightkurve: Kepler and TESS time series analysis in Python, Astrophysics Source Code Library (ascl:1812.013)

Luger R., Agol E., Kruse E., Barnes R., Becker A., ForemanMackey D., Deming D., 2016, AJ, 152, 100

Marshall P., et al., 2017, Lsst Science Collaborations Observing Strategy White Paper: "Science-Driven Optimization Of The Lsst Observing Strategy", doi:10.5281/zenodo.842713

Miknaitis G., et al., 2007, ApJ, 666, 674

Nicuesa Guelbenzu A., et al., 2012, A\&A, 548, A101

Olling R. P., et al., 2015, Nature, 521, 332

Pearson K. A., Palafox L., Griffith C. A., 2018, MNRAS, 474, 478

Poleski R., Penny M., Gaudi B. S., Udalski A., Ranc C., Barentsen G., Gould A., 2019, A\&A, 627, A54

Rest A., et al., 2014, The Astrophysical Journal, 795, 44

Rest A., et al., 2018, Nature Astronomy, 2, 307

Ricker G. R., et al., 2015, Journal of Astronomical Telescopes, Instruments, and Systems, 1, 014003

Ridden-Harper R., Tucker B. E., Sharp R., Gilbert J., Petkovic M., 2017, MNRAS, 472, 4521

Ridden-Harper R., et al., 2019, MNRAS, 490, 5551

Ridden-Harper R., Rest A., Narayan G., Barentsen G., in prep, The Astrophysical Journal, in prep

Rowan-Robinson M., 1999, Fireworks in a Dark Universe, 1, 3

Ryan G., van Eerten H., Piro L., Troja E., 2019, arXiv e-prints, p. arXiv:1909.11691

Scolnic D., et al., 2018, ApJ, 852, L3

Shappee B. J., et al., 2019, ApJ, 870, 13

Sharp R., Tucker B., Ridden-Harper R., Bloxham G., Petkovic M., 2016, in Proc. SPIE. p. 99080V, doi:10.1117/12.2231555

Smith K. W., et al., 2018, The Astronomer's Telegram, 11661, 1

Still M., Barclay T., 2012, PyKE: Reduction and analysis of Kepler Simple Aperture Photometry data, Astrophysics Source Code Library (ascl:1208.004)

Sullivan M., et al., 2011, ApJ, 737, 102

Tonry J. L., et al., 2018, PASP, 130, 064505

Vanderburg A., Johnson J. A., 2014, PASP, 126, 948

Varoquaux G., Buitinck L., Louppe G., Grisel O., Pedregosa F., Mueller A., 2015, GetMobile: Mobile Computing and Communications, 19, 29

Villar V. A., et al., 2017, ApJ, 851, L21

Vinícius Z., Barentsen G., Gully-Santiago M., Cody A. M., Hedges C., Still M., Barclay T., 2017, Ke- 
plerGO/PyKE, doi:10.5281/zenodo.835583, https:

//doi.org/10.5281/zenodo. 835583

Virtanen P., et al., 2019, arXiv e-prints, p. arXiv:1907.10121

Wenger M., et al., 2000, A\&AS, 143, 9

Zhu W., et al., 2017, Publications of the Astronomical Society of the Pacific, 129, 104501

\section{APPENDIX A: KEPLER/K2 ZEROPOINTS}

This paper has been typeset from a $\mathrm{T}_{\mathrm{E}} \mathrm{X} / \mathrm{LAT}_{\mathrm{E}} \mathrm{X}$ file prepared by the author. 
Table A1. Zeropoints for all Kepler/K2 detector channels. Channels with no values belong to the defunct modules 3,4 and 7 . The zerpoints are derived with data from Campaigns C01, C06, C12, C14, C16, and C17.

\begin{tabular}{|c|c|c|c|}
\hline Channel (0-42) & Zeropoint (0-42) & Channel (43-84) & Zeropoint (43-84) \\
\hline 1 & $25.27 \pm 0.06$ & 43 & $25.35 \pm 0.04$ \\
\hline 2 & $25.28 \pm 0.05$ & 44 & $25.31 \pm 0.05$ \\
\hline 3 & $25.23 \pm 0.16$ & 45 & $25.31 \pm 0.05$ \\
\hline 4 & $25.2 \pm 0.09$ & 46 & $25.31 \pm 0.04$ \\
\hline 5 & - & 47 & $25.3 \pm 0.04$ \\
\hline 6 & - & 48 & $25.28 \pm 0.05$ \\
\hline 7 & - & 49 & $25.27 \pm 0.05$ \\
\hline 8 & - & 50 & $25.26 \pm 0.05$ \\
\hline 9 & - & 51 & $25.19 \pm 0.04$ \\
\hline 10 & - & 52 & $25.2 \pm 0.07$ \\
\hline 11 & - & 53 & $25.29 \pm 0.04$ \\
\hline 12 & - & 54 & $25.28 \pm 0.05$ \\
\hline 13 & $25.29 \pm 0.09$ & 55 & $25.31 \pm 0.07$ \\
\hline 14 & $25.28 \pm 0.08$ & 56 & $25.26 \pm 0.07$ \\
\hline 15 & $25.23 \pm 0.08$ & 57 & $25.35 \pm 0.05$ \\
\hline 16 & $25.25 \pm 0.07$ & 58 & $25.36 \pm 0.04$ \\
\hline 17 & - & 59 & $25.35 \pm 0.05$ \\
\hline 18 & - & 60 & $25.33 \pm 0.05$ \\
\hline 19 & - & 61 & $25.32 \pm 0.04$ \\
\hline 20 & - & 62 & $25.3 \pm 0.04$ \\
\hline 21 & $25.4 \pm 0.07$ & 63 & $25.33 \pm 0.04$ \\
\hline 22 & $25.35 \pm 0.05$ & 64 & $25.3 \pm 0.04$ \\
\hline 23 & $25.34 \pm 0.04$ & 65 & $25.32 \pm 0.07$ \\
\hline 24 & $25.35 \pm 0.08$ & 66 & $25.38 \pm 0.05$ \\
\hline 25 & $25.33 \pm 0.05$ & 67 & $25.32 \pm 0.04$ \\
\hline 26 & $25.37 \pm 0.06$ & 68 & $25.35 \pm 0.09$ \\
\hline 27 & $25.31 \pm 0.06$ & 69 & $25.32 \pm 0.04$ \\
\hline 28 & $25.32 \pm 0.08$ & 70 & $25.33 \pm 0.05$ \\
\hline 29 & $25.27 \pm 0.1$ & 71 & $25.26 \pm 0.04$ \\
\hline 30 & $25.26 \pm 0.04$ & 72 & $25.23 \pm 0.04$ \\
\hline 31 & $25.24 \pm 0.04$ & 73 & $25.28 \pm 0.03$ \\
\hline 32 & $25.27 \pm 0.07$ & 74 & $25.32 \pm 0.04$ \\
\hline 33 & $25.27 \pm 0.04$ & 75 & $25.25 \pm 0.03$ \\
\hline 34 & $25.26 \pm 0.04$ & 76 & $25.27 \pm 0.05$ \\
\hline 35 & $25.19 \pm 0.05$ & 77 & $25.26 \pm 0.05$ \\
\hline 36 & $25.24 \pm 0.04$ & 78 & $25.25 \pm 0.04$ \\
\hline 37 & $25.37 \pm 0.04$ & 79 & $25.27 \pm 0.05$ \\
\hline 38 & $25.38 \pm 0.04$ & 80 & $25.24 \pm 0.03$ \\
\hline 39 & $25.36 \pm 0.06$ & 81 & $25.26 \pm 0.06$ \\
\hline 40 & $25.36 \pm 0.04$ & 82 & $25.26 \pm 0.05$ \\
\hline 41 & $25.33 \pm 0.05$ & 83 & $25.27 \pm 0.06$ \\
\hline 42 & $25.34 \pm 0.04$ & 84 & $25.26 \pm 0.05$ \\
\hline
\end{tabular}

Table A2. Kepler/K2 zeropoints from a selection of previous works.

\begin{tabular}{cc}
\hline Publication & Zeropoint \\
\hline Aigrain et al. (2015) & $25-25.3$ \\
Garnavich et al. (2016) & 25.47 \\
Zhu et al. (2017) & 25 \\
Dimitriadis et al. (2019) & $25.324 \pm 0.004$ \\
Poleski et al. (2019) & $25.47 \pm 0.13$ \\
\hline
\end{tabular}

